\title{
Improved bounds for the number of forests and acyclic orientations in the square lattice
}

\author{
N. Calkin \\ Department of Mathematical Sciences, \\ Martin Hall Box 341907, \\ Clemson, SC 29634-1907, USA. \\ calkin@math.clemson.edu \\ S. Noble \\ Department of Mathematical Sciences, \\ Brunel University \\ Kingston Lane, Uxbridge, UB8 3PH, U.K. \\ Steven.Derek.Noble@brunel.ac.uk
}

\author{
C. Merino \\ Instituto de Matemáticas, \\ Universidad Nacional de México. \\ Ciudad Universitaria, 04510, D.F. México. \\ merino@matem. unam.mx \\ M. Noy* \\ Dep. Matemàtica Aplicada II, \\ Universitat Politècnica de Catalunya \\ Pau Gargallo 5. 08028, Barcelona, Spain. \\ noy@ma2 .upc.es
}

Submitted: Oct 20, 2001; Accepted: Mar 25, 2002; Published: Jan 15, 2003

MR Subject Classifications: 05A16, 05C50

\begin{abstract}
In a recent paper Merino and Welsh (1999) studied several counting problems on the square lattice $L_{n}$. There the authors gave the following bounds for the asymptotics of $f(n)$, the number of forests of $L_{n}$, and $\alpha(n)$, the number of acyclic orientations of $L_{n}: 3.209912 \leq \lim _{n \rightarrow \infty} f(n)^{1 / n^{2}} \leq 3.84161$ and $22 / 7 \leq \lim _{n \rightarrow \infty} \alpha(n)^{1 / n^{2}} \leq$ 3.70925 .

In this paper we improve these bounds as follows: $3.64497 \leq \lim _{n \rightarrow \infty} f(n)^{1 / n^{2}} \leq$ 3.74101 and $3.41358 \leq \lim _{n \rightarrow \infty} \alpha(n)^{1 / n^{2}} \leq 3.55449$. We obtain this by developing a method for computing the Tutte polynomial of the square lattice and other related graphs based on transfer matrices.
\end{abstract}

\section{Introduction}

Given a graph $G=(V, E)$, a forest of $G$ is a subset $A$ of $E$ that contains no cycle. A spanning forest of $G$ is a spanning subgraph whose edge set is a forest. An acyclic orientation of $G$ is an assignment of a direction to every edge in $E$ such that there is no directed cycle. We denote by $\alpha(G)$ the number of acyclic orientations of $G$ and by $f(G)$ the number of spanning forests of $G$.

\footnotetext{
*Partially supported by projects SEUI-PB98-0933 and by CUR Gen. Cat. 1999SGR00356.
} 
In a recent paper Merino and Welsh [7] studied several counting problems on the square lattice $L_{n}$, the graph having as vertices the set $\{1, \ldots, n\} \times\{1, \ldots, n\}$ and where two vertices $(i, j)$ and $\left(i^{\prime}, j^{\prime}\right)$ are adjacent if $\left|i-i^{\prime}\right|+\left|j-j^{\prime}\right|=1$. Let $f(n)=f\left(L_{n}\right)$ and $\alpha(n)=\alpha\left(L_{n}\right)$ be the number of spanning forests and acyclic orientations, respectively, of $L_{n}$. It was shown in [7] that

$$
3.209912 \leq \lim _{n \rightarrow \infty} f(n)^{1 / n^{2}} \leq 3.84161,
$$

and that

$$
22 / 7 \leq \lim _{n \rightarrow \infty} \alpha(n)^{1 / n^{2}} \leq 3.70925 .
$$

In this paper we improve the above results by showing that

$$
3.64497 \leq \lim _{n \rightarrow \infty} f(n)^{1 / n^{2}} \leq 3.74101,
$$

and that

$$
3.41358 \leq \lim _{n \rightarrow \infty} \alpha(n)^{1 / n^{2}} \leq 3.55449 .
$$

Our interest in computing $\alpha(n)$ and $f(n)$ is mainly because of the importance of the square lattice in statistical physics, but we also refered the reader to the discussion about counting problems on the square lattice in the introduction of [7].

It is important to mention that $\alpha(G)$ and $f(G)$ have been proved \#P-hard for planar bipartite graphs [11] and more recently for the class of grid graphs of maximum degree 3 [12], where a graph $G$ is a grid graph if it is a subgraph of the square lattice $L_{n}$ for some $n$. This last result implies that computing $\alpha(G)$ and $f(G)$ is \#P-hard for the class of grid graphs to which the square lattice $L_{n}$ belongs. So, computing $\alpha(n)$ or $f(n)$ depends on properties of the family $\left\{L_{n} \mid n \geq 2\right\}$.

The key tool for proving our results is a method for computing the Tutte polynomial of square lattices and other related graphs based on transfer matrices. The method is interesting in itself and has been usefully applied to other families of graphs [8].

We describe the method in Section 3, after a short introduction to the Tutte polyonmial in Section 2. In Section 4 we explain how to evaluate the Tutte polynomial at particular points using the transfer-matrix approach. Then in Sections 5 and 6 we prove the main results of the paper, namely the bounds (1.1) and (1.2). We conclude with one additional result.

The use of transfer-matrices is common in enumeration problems dealing with square lattices (see $[2,5]$ ) but our approach is novel for computing Tutte polynomials. Let us mention that a different transfer-matrix approach is used in [1] for computing chromatic polynomials of square lattices.

\section{The Tutte polynomial}

Let $G=(V, E)$ be a graph with vertex set $V$ and edge set $E$ (loops and multiple edges are allowed). For every subset $A \subseteq E$, its rank is $r(A)=|V|-\omega(A)$, where $\omega(A)$ is the 
number of connected components of the spanning subgraph $(V, A)$. The rank polynomial of $G$ is defined as

$$
R(G ; x, y)=\sum_{A \subseteq E} x^{r(E)-r(A)} y^{|A|-r(A)}
$$

The Tutte polynomial of $G$ is obtained from the rank polynomial by a simple change of variables:

$$
T(G ; x, y)=R(G ; x-1, y-1) .
$$

The Tutte polynomial contains much information on the graph $G$; we refer to [7] and the survey paper [3] for background information. In particular:

- $T(G ; 2,1)$ is the number $f(G)$ of spanning forests in $G$;

- $T(G ; 2,0)$ is the number $\alpha(G)$ of acyclic orientations in $G$.

In the last section we also need the following, where an orientation is totally cyclic if every edge is contained in some directed cycle and we consider $G$ to be connected.

- $T(G ; 1,2)$ is the number of spanning connected subgraphs in $G$;

- $T(G ; 0,2)$ is the number of totally cyclic orientations in $G$.

In order to simplify the computations in the next sections, we work with the rank polynomial instead of the Tutte polynomial; this poses no problem since $T(G ; 2,1)=$ $R(G ; 1,0)$, and so on.

Unless otherwise indicated, all subgraphs of a given lattice are considered to be spanning.

\section{A transfer-matrix approach}

We see from the previous section that the task of computing $f(G)$ and $\alpha(G)$ amounts to the evaluation of the Tutte polynomial of $G$ at the points $(2,1)$ and $(2,0)$. However, as mentioned before, the evaluation of the Tutte polynomial at these points is \#P-hard for planar bipartite graphs [11] and even for grid graphs of maximum degree 3 [12].

The approach for obtaining the bounds in Sections 5 and 6 is to subdivide a large lattice into smaller (not necessarily square) sublattices. This motivates the following definition: $L_{n, m}$ is the $n \times m$ lattice, that is, the graph having vertices $\{1, \ldots, n\} \times\{1, \ldots, m\}$ in which two vertices $(i, j)$ and $\left(i^{\prime}, j^{\prime}\right)$ are adjacent if $\left|i-i^{\prime}\right|+\left|j-j^{\prime}\right|=1$. According to the notation of the introduction, we have that $L_{n}=L_{n, n}$.

For the Tutte polynomial we have the contraction-deletion formula (see [3]),

$$
T(G ; x, y)=T(G-e ; x, y)+T(G / e ; x, y),
$$

where e is any edge of $\mathrm{G}$ which is not a loop or a bridge. If $e$ is a loop we have that

$$
T(G ; x, y)=y T(G-e ; x, y)
$$


and if $e$ is a bridge, we have that

$$
T(G ; x, y)=x T(G / e ; x, y) .
$$

Then, for small values of $m$, one can obtain a linear recurrence for the family of polynomials $\left\{T\left(L_{n, m} ; x, y\right)\right\}_{n \geq 0}$ and solve it directly. But already in the case $m=3$ this is very cumbersome.

Our strategy instead consists in viewing the lattice $L_{n, m}$ as the union of $L_{n-1, m}$ and a comb graph $P_{m}$, which is just $L_{2, m}$ with the edges in the first column deleted (see Fig. 1 ).
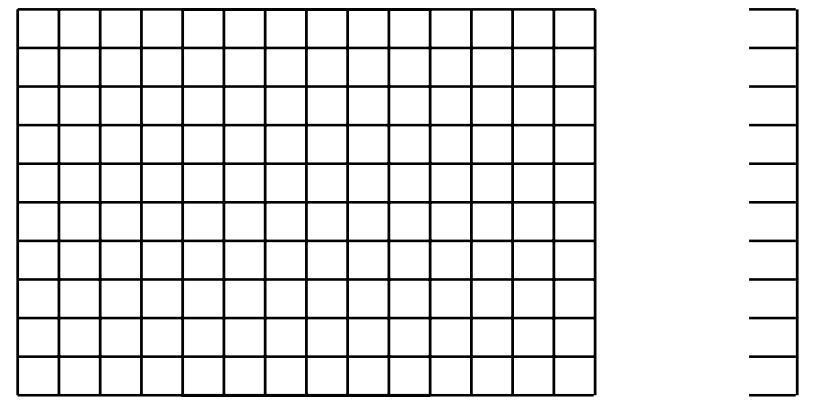

Figure 1: The lattice $L_{15,11}$ and the 11-comb graph $P_{11}$.

Consider now the formula (2.1) when $G=L_{n, m}$. Each $A \subseteq E\left(L_{n, m}\right)$ can be written as

$$
A=B \cup C, \quad \text { with } B \subseteq E\left(L_{n-1, m}\right), \quad C \subseteq E\left(P_{m}\right),
$$

and clearly, $|A|=|B|+|C|$. Let us write

$$
r(B \cup C)=r(B)+\delta(B, C),
$$

where $\delta(B, C)$ is the increment in the rank of $B$ produced by the addition of $C$. Then we rewrite $(2.1)$ as

$$
\begin{aligned}
R\left(L_{n, m} ; x, y\right) & =x^{r\left(L_{n, m}\right)} \sum_{A=B \cup C} x^{-r(A)} y^{|A|-r(A)} \\
& =x^{r\left(L_{n, m}\right)} \sum_{\substack{B \subseteq E\left(L_{n-1, m}\right) \\
C \subseteq E\left(P_{m}\right)}} x^{-r(B)} y^{|B|-r(B)}\left(\sum_{C} x^{-\delta(B, C)} y^{|C|-\delta(B, C)}\right) .
\end{aligned}
$$

In order to use this formulation in a recursive scheme we must be able to compute the increment $\delta(B, C)$ without knowledge of the whole edge-set $B$. Given an edge-set $B$, we label the $m$ vertices in the $(n-1)$-th column according to the component of the spanning subgraph induced by $B$ to which they belong; the components are labeled canonically $1,2, \ldots$ as they appear. In this way we get a state $\sigma(B)=\left(s_{1}, \ldots, s_{m}\right)$. An example is given in Fig. 2.

Then the following lemma is clear, since from the knowledge of $\sigma(B)$ we can update the number of components in the union $B \cup C$. In the example in Fig. 2 we have $r(B)=$ $26, r(C)=5$ and $\delta(B, C)=4$. 


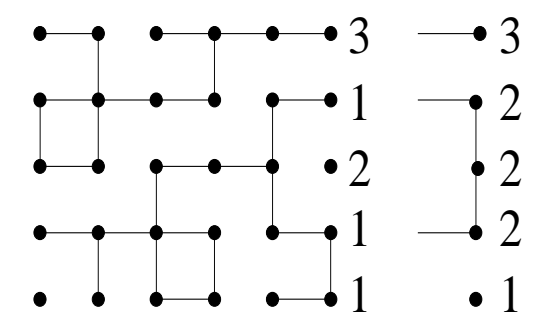

Figure 2: The state $\sigma(B)=(1,1,2,1,3)$ and $\sigma(B \cup C)=(1,2,2,2,3)$.

Lemma 3.1. The rank of $B \cup C$, and hence $\delta(B, C)$, can be computed from the knowledge of the state $\sigma(B)$ and $C$.

Proof. For the state $\sigma(B)=\left(s_{1}, \ldots, s_{m}\right)$ and a subset of edges $C$ of $P_{m}$ we construct a graph with vertices $\{1,2\} \times\{1, \ldots, m\}$ and edges $B^{\prime} \cup C$, where $B^{\prime}$ is the set of edges joining $(1, i)$ with $(1, j)$ for each pair $i, j$ such that $s_{i}=s_{j}$. Call this graph $G_{\sigma(B), C}$.

Now it is not difficult to check that

$$
-\delta(B, C)=\omega\left(G_{\sigma(B), C}\right)-|\sigma(B)|-m,
$$

where $|\sigma(B)|$ is the number of components of $\sigma(B)$.

If in the subgraph induced by $B$, the last $m$ vertices are in $k$ different components, then $\sigma(B)$ induces a partition $\pi$ of $[m]=\{1, \ldots, m\}$ into $k$ blocks. This must be a noncrossing partition: there do not exist two blocks $\beta$ and $\beta^{\prime}$ of $\pi$ and elements $a<b<c<d$ such that $a, c \in \beta$ and $b, d \in \beta^{\prime}$. From now on we use state and partition indistinctly. If we denote by $\mathcal{N C}_{m}$ the set of all non-crossing partitions of $[m]$, then it is well-known [10] that $\left|\mathcal{N C}_{m}\right|=c_{m}$, where

$$
c_{m}=\frac{1}{m+1}\left(\begin{array}{c}
2 m \\
m
\end{array}\right)
$$

is a Catalan number. The total number of partitions, a Bell number, is much larger.

For fixed $m$, we define a $c_{m} \times c_{m}$ matrix $\Lambda_{m}$ as follows. The rows and columns are indexed by the non-crossing partitions of $[m]$ ordered lexicographically. The entries of $\Lambda_{m}$ are initially set to 0 . Let $\sigma=\left(s_{1}, \ldots, s_{m}\right)$ be any non-crossing partition of $[m]$, and let $C$ be any subset of the $m$-comb $P_{m}$. Consider $\sigma$ as the state of a subset $B$ of edges in the lattice $L_{n-1, m}$, add the edge-set $C$, and compute $\delta(B, C)$ and the new state $\sigma^{\prime}=\sigma(B \cup C)$. Then add the term

$$
x^{-\delta(B, C)} y^{|C|-\delta(B, C)}
$$

to the $\left(\sigma, \sigma^{\prime}\right)$ entry of $\Lambda_{m}$. In order to illustrate the procedure we show below the computations when $m=2$ and $\sigma=(1,1)$. In the table, $e$ and $g$ are the two horizontal edges of $P_{2}$, and $f$ is the vertical edge. 


\begin{tabular}{|ccccc|c|}
\hline Initial state & $C$ & $|C|$ & $\delta(B, C)$ & Final state & Contribution to $\Lambda_{2}$ \\
\hline$(1,1)$ & $\emptyset$ & 0 & 0 & $(1,2)$ & 1 \\
$(1,1)$ & $\{e\}$ & 1 & 1 & $(1,2)$ & $(x y)^{-1} y$ \\
$(1,1)$ & $\{f\}$ & 1 & 1 & $(1,1)$ & $(x y)^{-1} y$ \\
$(1,1)$ & $\{g\}$ & 1 & 1 & $(1,2)$ & $(x y)^{-1} y$ \\
$(1,1)$ & $\{e, f\}$ & 2 & 2 & $(1,1)$ & $(x y)^{-2} y^{2}$ \\
$(1,1)$ & $\{f, g\}$ & 2 & 2 & $(1,1)$ & $(x y)^{-2} y^{2}$ \\
$(1,1)$ & $\{e, g\}$ & 2 & 2 & $(1,1)$ & $(x y)^{-2} y^{2}$ \\
$(1,1)$ & $\{e, f, g\}$ & 3 & 2 & $(1,1)$ & $(x y)^{-2} y^{3}$ \\
\hline
\end{tabular}

Similar computations when $\sigma=(1,2)$ give the final value

$$
\Lambda_{2}=\left(\begin{array}{ll}
x^{-1}+3 x^{-2}+y x^{-2} & 1+2 x^{-1} \\
x^{-1}+2 x^{-2}+x^{-3} & 1+2 x^{-1}+x^{-2}
\end{array}\right) .
$$

Next, we define a vector $X_{m}$ of length $c_{m}$, indexed by the non-crossing partitions $\sigma$ of $[m]$ as in the case of $\Lambda_{m}$. For every edge-set $B$ of $L_{1, m}$ (which is just a path of length $m-1$ ), let $\sigma(B)$ be its state as before. We say that a partition $\tau$ is realizable if there exists $B \subseteq L_{1, m}$ with $\sigma(B)=\tau$. In this case $B$ is a realization of $\tau$. Notice that if a realization exists, then it is unique. Also, only those $\tau$ which are non-decreasing are realizable; for instance, $(1,2,1)$ is not.

We are ready for the definition of $X_{m}$ and for the main result in this section.

$$
\left(X_{m}\right)_{\tau}= \begin{cases}x^{-|B|} & \text { if } \tau \text { has realization } B \\ 0 & \text { otherwise }\end{cases}
$$

Theorem 3.2. For integers $n, m \geq 2$, we have

$$
R\left(L_{n, m} ; x, y\right)=x^{n m-1} X_{m}^{t} \cdot\left(\Lambda_{m}\right)^{n-1} \cdot \overrightarrow{1}
$$

where $X_{m}$ is the vector of length $c_{m}$ defined above, and $\overrightarrow{1}$ is the vector of length $c_{m}$ with all entries equal to 1.

Proof. By definition, the vector $X_{m}$ encodes the contribution to the rank polynomial of the edges of the first column $L_{1, m}$ of $L_{n, m}$. Every time we multiply by $\Lambda_{m}$ we are adding the contribution of the edges of a comb graph $P_{m}$. Finally, multiplying by $\overrightarrow{1}$ we sum up all the contributions from all possible states. 
Continuing with the previous example, it follows that

$$
R\left(L_{n, 2} ; x, y\right)=x^{2 n-1}\left(x^{-1}, 1\right) \cdot\left(\Lambda_{2}\right)^{n-1} \cdot \overrightarrow{1},
$$

where $\Lambda_{2}$ is as before. Substituting $x, y$ for $x-1, y-1$ one gets the Tutte polynomial of $L_{n, 2}$. Using this formula, the reader can check, for example, that

$$
T\left(L_{3,2} ; x, y\right)=2 x^{2}+x+2 x y+y+y^{2}+3 x^{3}+2 x^{2} y+2 x^{4}+x^{5} .
$$

\section{Numerical values}

In principle, the above method can be used to compute the Tutte polynomial of the lattice $L_{n, m}, n, m \geq 2$, but computationally it is not feasible, as the required space to store the transfer-matrix grows exponentially. As an example, for $m=10$ the transfer-matrix is a 16792-by-16792 matrix. Even for small values of $n$ and $m$, the above computation involves storing large polynomials for each entry of the transfer-matrix, so that although possible, it is very cumbersome. Another possibility is to evaluate the polynomial at a sufficient number of points and then interpolate. This option is more practical, but we have not explored it.

However, for some small values of $n$ and $m$ we can evaluate the Tutte polynomial at particular points easily. By Theorem 3.2, to evaluate $T\left(L_{n, m} ; x_{0}+1, y_{0}+1\right)$, we just have to evaluate

$$
x_{0}^{n m-1} \hat{X}_{m}^{t} \cdot \hat{\Lambda}_{m} \cdot \overrightarrow{1}
$$

where $\hat{X}_{m}$ and $\hat{\Lambda}_{m}$ are the vector and matrix respectively, defined in the last section, with the substitutions $x=x_{0}$ and $y=y_{0}$.

We have written $\mathrm{C}$ programs indices.c and matrix.c which can compute the matrix $\hat{\Lambda}_{m}$ at any given point. We also have a program called vector.c that can compute the vector $\hat{X}_{m}$ at $x=x_{0}$. ${ }^{1}$

Using this procedure with the values $\left(x_{0}, y_{0}\right)=(1,0)$ and $\left(x_{0}, y_{0}\right)=(1,-1)$, we compute $f(n)$ and $\alpha(n)$ for $2 \leq n \leq 7$. The values are shown in Table 1 .

The values for $f(7)$ and $\alpha(7)$ can be used to improve the upper bound given in [7] by using Theorem 6.1 and Theorem 5.4 from the same paper, obtaining the bounds

$$
\begin{aligned}
& \lim _{n \rightarrow \infty}(f(n))^{1 / n^{2}} \leq 3.78649853538319 \ldots \\
& \lim _{n \rightarrow \infty}(\alpha(n))^{1 / n^{2}} \leq 3.62330970816373 \ldots
\end{aligned}
$$

\section{Upper bounds}

The procedure described in Section 4 allows us to actually compute the number of forests of $L_{n, m}$, which from now on we denote by $f(n, m)$, for a fixed $m$ and an arbitrary $n$.

\footnotetext{
${ }^{1}$ These programs can be obtained in http://calli.matem. unam.mx/ merino/publications.html.
} 
Forests and acyclic orientations

\begin{tabular}{|c|c|c|}
\hline Side $n$ & Number of forests & Number of acyclic orientations \\
\hline \hline 2 & 15 & 14 \\
3 & 3102 & 2398 \\
4 & 8790016 & 5015972 \\
5 & $3.410086174080000 e+11$ & $1.280914342660000 \mathrm{e}+11$ \\
6 & $1.810755082420676 e+17$ & $3.993185613821266 \mathrm{e}+16$ \\
7 & $1.315927389374152 e+24$ & $1.519663682749935 \mathrm{e}+23$ \\
\hline
\end{tabular}

Table 1: This table displays the values of $f(n)$ and $\alpha(n)$ for $1 \leq n \leq 7$.

In this section we denote by $A_{m}$ the matrix $\Lambda_{m}$ evaluated when $x=1, y=0$. To compute $f(n, m)$ we have to evaluate $\left.X_{m}^{t}\right|_{x=1} A_{m}^{n-1} \overrightarrow{1}$, where the vector $\left.X_{m}\right|_{x=1}$ has just 0-1 entries.

The first observation is that $a^{t} A \overrightarrow{1} \leq\|A\|_{1}$, where $a$ is a $0-1$ vector, $A$ is a $k \times k$ real matrix and $\|\cdot\|_{1}$ is the $l_{1}$ matrix norm, that is $\|A\|_{1}=\sum_{i} \sum_{j}\left|A_{i j}\right|$.

Secondly, the following is a well known result in linear algebra (see, for example, $[6$, Corollary 5.6.14]).

Theorem 5.1. Let $\|\cdot\|$ be a matrix norm on $\mathcal{M}_{k}$, the $k \times k$ real matrices. Then, for $A \in \mathcal{M}_{k}$,

$$
\rho(A)=\lim _{k \rightarrow \infty}\left\|A^{k}\right\|^{1 / k},
$$

where $\rho(A)=\max \{|\lambda| \mid \lambda$ is an eigenvalue of $A\}$ is the spectral radius of $A$.

Combining these two results we obtain the following theorem.

Theorem 5.2. For any fixed natural number $m$,

$$
\lim _{n \rightarrow \infty} f(n, m)^{1 / n} \leq \rho\left(A_{m}\right) .
$$

This upper bound has a direct implication on $\lim _{n \rightarrow \infty} f(n)^{1 / n^{2}}$, as we prove in the following theorem.

Theorem 5.3. For $k \geq 1$,

$$
\lim _{n \rightarrow \infty} f(n)^{1 / n^{2}} \leq 2^{1 / k}\left(\rho\left(A_{k}\right)\right)^{1 / k} .
$$

Proof. Let $k$ be a fixed integer. From a square lattice of side $k p$ we select $p$ lattices $L_{k p, k}$, whose bottom left-hand corners are the points $(1, k i+1)$, with $0 \leq i \leq p-1$. Call this set of subgraphs $\mathcal{C}$.

Choose for every subgraph in $\mathcal{C}$ a spanning forest and then choose any subset of the remaining $(p-1) k p$ edges in $L_{k p}$. Any spanning forest of $L_{k p}$ can be obtained in this way, but this is clearly an over counting, so we conclude that

$$
f(k p) \leq 2^{(p-1) k p}(f(k, k p))^{p} \leq 2^{k p^{2}}(f(k, k p))^{p} .
$$


Hence

$$
f(k p)^{1 /(k p)^{2}} \leq 2^{1 / k} f(k, k p)^{1 / k^{2} p} .
$$

By taking the limit as $p \rightarrow \infty$ we get the result using Theorem 5.2.

Using matlab, we compute the values of $\rho\left(A_{m}\right)$ for $2 \leq m \leq 8$, once we have generated the matrix $A_{m}$ with the programs indices.c and matrix.c. As $m$ is increased the upper bound gets tighter, so using the best value obtained we have the following

\section{Corollary 5.4.}

$$
\lim _{n \rightarrow \infty} f(n)^{1 / n^{2}} \leq 3.74100178268615 .
$$

We now turn to acyclic orientations. If we denote by $A_{m}^{\prime}$ the matrix $\left.\Lambda_{m}\right|_{\substack{x=1 \\ y=-1}}$, we can follow steps similar to those above and obtain a result similar to Theorem 5.3 but for the number of acyclic orientations of $L_{n}$.

Theorem 5.5. For $k \geq 1$,

$$
\lim _{n \rightarrow \infty} \alpha(n)^{1 / n^{2}} \leq 2^{1 / k}\left(\rho\left(A_{k}^{\prime}\right)\right)^{1 / k} .
$$

Now, the best value that we manage to compute is for $\rho\left(A_{8}^{\prime}\right)$, and this gives us the following

\section{Corollary 5.6.}

$$
\lim _{n \rightarrow \infty} \alpha(n)^{1 / n^{2}} \leq 3.55448520960037 .
$$

Corollaries 5.4 and 5.6 give improvements on the upper bounds of previous results [7] and on the ones just mentioned in the last section.

Note. By using first-order perturbation estimates the above results obtained by matlab can be considered correct up to the last decimal.

\section{Lower bounds}

In the previous section we used the transfer-matrix method to improve the upper bounds given in [7]. In this section we improve the lower bounds of the same reference.

We define the $n, k$-fan graph $\mathcal{F}_{n}^{k}, k \geq 1$, as the graph with vertex set $\{\hat{0}\} \cup\{1, \ldots, n\} \times$ $\{1, \ldots, k\}$. There is an edge between vertices $(i, j)$ and $\left(i^{\prime}, j^{\prime}\right)$ if $\left|i-i^{\prime}\right|+\left|j-j^{\prime}\right|=1$; also we have all the edges $\hat{0} \sim(i, 1)$, for $1 \leq i \leq n$ (see Figure 3 ). The reader may find it helpful to think that for a fixed $k$, increasing $n$ will make $\mathcal{F}_{n}^{k}$ grow to the right.

For the proofs of the following two theorems one more definition is required. We define the $n, k$-comb graph $P_{n}^{k}$ to be the graph with vertex set $\{1, \ldots, n\} \times\{0, \ldots, k\}$. There is an edge between vertices $(i, j),\left(i^{\prime}, j^{\prime}\right)$ if $\left|i-i^{\prime}\right|+\left|j-j^{\prime}\right|=1$, whenever $j>0$; also we have all the edges $(i, 0) \sim(i, 1), i \in\{1, \ldots, n\}$. Note that there is a natural bijection from the set of edges of $P_{n}^{k}$ to the set of edges of $\mathcal{F}_{n}^{k}$. 

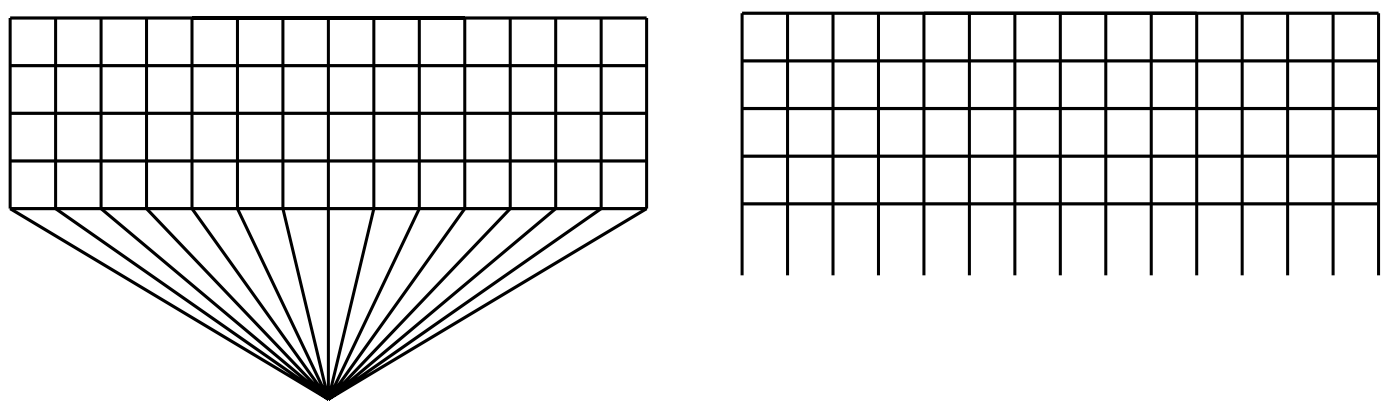

Figure 3: The 15,5-fan graph $\mathcal{F}_{15}^{5}$ and 15,5-comb graph $P_{15}^{5}$.

Theorem 6.1. For an arbitrary but fixed integer $k$,

$$
\left(\lim _{n \rightarrow \infty}\left(f\left(\mathcal{F}_{n}^{k}\right)\right)^{1 / n}\right)^{1 / k} \leq \lim _{n \rightarrow \infty} f(n)^{1 / n^{2}} .
$$

Proof. From a square lattice of side $k p+1$ we select $p$ different $k p+1, k$-comb graphs, $G_{i}, 0 \leq i \leq p-1$, whose bottom left-hand corners are the points $(1, k i+1)$. Call this set of subgraphs $\mathcal{C}$. Observe that there are $k p$ edges left at the bottom of $L_{k p+1}$ that do not belong to any of the $G_{i}$ 's.

Choose one spanning forest $B_{i}^{\prime}$ in $\mathcal{F}_{k p+1}^{k}$ for every subgraph $G_{i}$ in $\mathcal{C}$, and take the edges in $G_{i}$ that correspond (under the natural bijection) to this forest, say $B_{i}$, with $0 \leq i \leq p-1$.

The set of edges $B=\bigcup_{i=0}^{p-1} B_{i}$ corresponds to the edge set of a spanning forest of $L_{k p+1}$. The reason is the following. Suppose there is a cycle $C$ in $B$, then it would intersect some of the subgraphs $B_{i}$. The cycle $C$ cannot be inside an element $B_{i}$, as this would contradict our choice of $B_{i}^{\prime}$. Let $j_{0}$ be the maximum $j$ such that $B_{j}$ intersects $C$. Thus, $B_{j_{0}}$ contains a path from $\left(l, j_{0} k+1\right)$ to $\left(h, j_{0} k+1\right)$ for some $1 \leq l, h \leq k p+1$. This path in $B_{j_{0}}$ maps onto a cycle in $\mathcal{F}_{k p+1}^{k}$ that passes through $\hat{0}$ and this contradicts our choice of $B_{j_{0}}^{\prime}$. Therefore there is no such cycle $C$.

Any such choice of the $B_{i}^{\prime}, 0 \leq i \leq p-1$ will give a different spanning forest of $L_{p k+1}$, So

$$
\left(f\left(\mathcal{F}_{k p+1}^{k}\right)\right)^{p} \leq f(k p+1)
$$

Then

$$
\left(\left(f\left(\mathcal{F}_{k p+1}^{k}\right)\right)^{\frac{1}{p k+1}}\right)^{\frac{p}{k p+1}} \leq(f(k p+1))^{\frac{1}{(p k+1)^{2}}}
$$

and by taking the limit as $p \rightarrow \infty$ we get the result.

In the same way, an acyclic orientation on a fan graph induces an acyclic orientation on the corresponding comb graph. A simple adaptation of the last proof also gives a proof of the following 
Theorem 6.2. For an arbitrary but fixed integer $k$,

$$
\left(\lim _{n \rightarrow \infty}\left(\alpha\left(\mathcal{F}_{n}^{k}\right)\right)^{1 / n}\right)^{1 / k} \leq \lim _{n \rightarrow \infty} \alpha(n)^{1 / n^{2}}
$$

As an application consider the sequence $\left\{\alpha\left(\mathcal{F}_{n}^{2}\right)\right\}_{n=1}^{\infty}$. By using the contraction-deletion formulas (3.1), (3.2) and (3.3), we get the following recurrence relation

$$
\alpha\left(\mathcal{F}_{n}^{2}\right)=13 \alpha\left(\mathcal{F}_{n-1}^{2}\right)-27 \alpha\left(\mathcal{F}_{n-2}^{2}\right),
$$

with the initial conditions $\alpha\left(\mathcal{F}_{1}^{2}\right)=4$ and $\alpha\left(\mathcal{F}_{2}^{2}\right)=42$. By solving this recurrence we get

$$
\alpha\left(\mathcal{F}_{n}^{2}\right)=c_{1}\left(\frac{13+\sqrt{61}}{2}\right)^{n}+c_{2}\left(\frac{13-\sqrt{61}}{2}\right)^{n},
$$

where $c_{1} \approx 0.3890957718$ and $c_{2} \approx 0.01872540139$. Then, by Theorem 6.2 we obtain

$$
\lim _{n \rightarrow \infty} \alpha(n)^{1 / n^{2}} \geq \sqrt{\frac{13+\sqrt{61}}{2}}=3.225697574 .
$$

In principle, we could find recurrence relations for the sequences $f\left(\mathcal{F}_{n}^{k}\right)$ and $\alpha\left(\mathcal{F}_{n}^{k}\right)$ for a fixed $k>1$, using contraction and deletion. Then, by solving the recurrence, we obtain an explicit expression for these sequences. This is, however, very cumbersome. Already for $k=3$ we have to express $f\left(\mathcal{F}_{n}^{3}\right)$ as a solution of several linear recurrence relations with many variables.

Again, we choose to use the transfer-matrix method already developed and compute

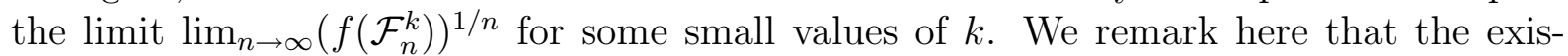
tance of $\lim _{n \rightarrow \infty}\left(f\left(\mathcal{F}_{n}^{k}\right)\right)^{1 / n}$ and $\lim _{n \rightarrow \infty}\left(\alpha\left(\mathcal{F}_{n}^{k}\right)\right)^{1 / n}$ will be a consequence of Theorems 6.12 and 6.13 .

Let $H=\left\{e_{1}, \ldots, e_{n-1}\right\}$ be the set of edges $e_{1}=(1,1) \sim(2,1), \ldots, e_{n-1}=(n-$ $1,1) \sim(n, 1)$. Clearly, the graphic matroid $M\left(\mathcal{F}_{n}^{k-1}\right)$ is isomorphic to the graphic matroid $M\left(L_{n, k} / H\right)$, where $L_{n, k} / H$ is the graph $L_{n, k}$ with the edges in $H$ contracted. Even more, after a relabeling of the vertices, we can consider their ground sets to be the same. For example, the identified vertices in $M\left(L_{n, k} / H\right)$ can be considered to be $\hat{0}$, the same vertex as in $\mathcal{F}_{n}^{k-1}$, so, we consider here $E\left(\mathcal{F}_{n}^{k-1}\right)=E\left(L_{n, k} / H\right)$. If $r$ denotes the rank function of $M\left(L_{n, k}\right)$ and $r^{\prime}$ denotes the rank function of $M\left(\mathcal{F}_{n}^{k-1}\right)$ for a set $B \subseteq E\left(\mathcal{F}_{n}^{k-1}\right)$, these functions are related by

$$
\begin{aligned}
r^{\prime}(B) & =r\left(B \cup\left\{e_{1}, \ldots, e_{n-1}\right\}\right)-r\left(\left\{e_{1}, \ldots, e_{n-1}\right\}\right) \\
& =r\left(B \cup\left\{e_{1}, \ldots, e_{n-1}\right\}\right)-(n-1),
\end{aligned}
$$

where the first equality is a well known property of the rank function (see $[9$, Proposition 3.1.6]). In particular

$$
r^{\prime}\left(E\left(\mathcal{F}_{n}^{k-1}\right)\right)=r\left(E\left(L_{n, k}\right)\right)-(n-1) .
$$


The definition of the Rank polynomial gives for $M\left(\mathcal{F}_{n}^{k-1}\right)$ that

$$
R\left(\mathcal{F}_{n}^{k-1} ; x, y\right)=\sum_{B \subseteq E\left(\mathcal{F}_{n}^{k-1}\right)} x^{r^{\prime}\left(E\left(\mathcal{F}_{n}^{k-1}\right)\right)-r^{\prime}(B)} y^{|B|-r^{\prime}(B)} .
$$

If for each $B \subseteq E\left(\mathcal{F}_{n}^{k-1}\right)$ we take $A=B \cup\left\{e_{1}, \ldots, e_{n-1}\right\}$, then, by equations (6.1) and (6.2), for a particular $B$, the exponent of $x$ in (6.3) equals

$$
r\left(E\left(L_{n, m}\right)\right)-(n-1)-r(A)+(n-1)=r\left(E\left(L_{n, m}\right)\right)-r(A)
$$

and the exponent of $y$ equals

$$
|A|-(n-1)-r(A)+(n-1)=|A|-r(A) .
$$

So, we obtain

$$
R\left(\mathcal{F}_{n}^{k-1} ; x, y\right)=\sum_{\substack{A=B \cup\left\{e_{1}, \ldots, e_{n-1}\right\} \\ B \subseteq E\left(\mathcal{F}_{n}^{k-1}\right)}} x^{r\left(E\left(L_{n, k}\right)\right)-r(A)} y^{|A|-r(A)} .
$$

The same procedure as in Section 3 can be used to compute the expresion (6.4) with the only restriction that the edge set $C \subseteq E\left(P_{k}\right)$ has to contain the edge joining vertices $(1,1)$ and $(2,1)$. We denote by $\Lambda_{k}^{\prime}$ the corresponding matrix for this case. Then the analogue to Theorem 3.2 is the following.

Theorem 6.3. For $k, n \geq 2$ two integers we have

$$
R\left(\mathcal{F}_{n}^{k-1} ; x, y\right)=x^{k n-1} X_{k}^{t}\left(\Lambda_{k}^{\prime}\right)^{n-1} \overrightarrow{1},
$$

where $X_{k}$ and $\overrightarrow{1}$ are as in Theorem 3.2.

We illustrate the procedure by constructing the row corresponding to state $(1,1)$ in $\Lambda_{2}^{\prime}$. In the table, $e$ is the horizontal edge incident to vertices $(1,1)$ and $(1,2), g$ is the other horizontal edge of $P_{2}$, and $f$ is the vertical edge. (Beware of confusion between a vertex and a state).

\begin{tabular}{|ccccc|c|}
\hline Initial state & $C$ & $|C|$ & $\delta(B, C)$ & Final state & Contribution to $\Lambda_{2}$ \\
\hline$(1,1)$ & $\{e\}$ & 1 & 1 & $(1,2)$ & $(x y)^{-1} y$ \\
$(1,1)$ & $\{e, f\}$ & 2 & 2 & $(1,1)$ & $(x y)^{-2} y^{2}$ \\
$(1,1)$ & $\{e, g\}$ & 2 & 2 & $(1,1)$ & $(x y)^{-2} y^{2}$ \\
$(1,1)$ & $\{e, f, g\}$ & 3 & 2 & $(1,1)$ & $(x y)^{-2} y^{3}$ \\
\hline
\end{tabular}

Similar computations when $\sigma=(1,2)$ give the final value

$$
\Lambda_{2}=\left(\begin{array}{ll}
2 x^{-2}+y x^{-2} & x^{-1} \\
x^{-2}+x^{-3} & x^{-1}+x^{-2}
\end{array}\right) .
$$


The example shows that any entry of $\Lambda_{k}^{\prime}$ is formed by a subset of the terms in the corresponding entry of $\Lambda_{k}$.

As we are interested in $\lim _{n \rightarrow \infty}\left(f\left(\mathcal{F}_{n}^{k-1}\right)\right)^{1 / n}$ and $\lim _{n \rightarrow \infty}\left(\alpha\left(\mathcal{F}_{n}^{k-1}\right)\right)^{1 / n}$, we consider here just the evaluations $x_{0}=1$ and $y_{0}=0$ or $y_{0}=-1$. We denote for the rest of this section the square real matrix $\left.\Lambda_{k}^{\prime}\right|_{\substack{x=1 \\ y=0}}$ by $D_{k}$; the matrix $\left.\Lambda_{k}^{\prime}\right|_{\substack{x=1 \\ y=-1}}$ by $D_{k}^{\prime}$; and the column vector $\left.X_{k}\right|_{x=1}$ by $a_{k}$.

In contrast with the analysis in the previous section, where a simple observation on the $l_{1}$ norm was sufficient to get the upper bounds, to obtain lower bounds we need to

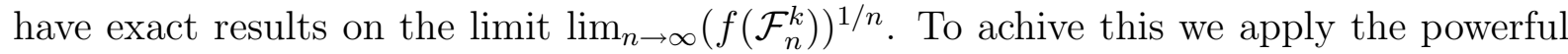
Perron-Frobenius Theorem to these matrices, but we need some other results first.

Definition 6.4. The directed graph of an $m \times m$ real matrix $A$, denoted by $\Gamma(A)$, is the directed graph on $m$ nodes $c_{1}, \ldots, c_{m}$ such that there is a directed arc in $\Gamma(A)$ from $c_{i}$ to $c_{j}$ if and only if $(A)_{i j} \neq 0$.

Then there is the following well known theorem (see [6, Theorem 6.2.24]).

Theorem 6.5. Let $A$ be an $m \times m$ nonnegative real matrix. Then $A$ is irreducible if and only if $\Gamma(A)$ is strongly connected.

Before continuing, we introduce some notation. For $\sigma(B)=\sigma_{1}$ and $\sigma_{2}$, two states, we say $\sigma_{1} \stackrel{C}{\longrightarrow} \sigma_{2}$, if there exists $C \subseteq E\left(P_{k}\right)$ such that $\sigma(B \cup C)=\sigma_{2}$.

With this notation and formula (3.4) the construction of the matrix $\Lambda_{k}$ in Section 3 can be described as follows: in entry $\left(\sigma_{1}, \sigma_{2}\right)$ we add the term

$$
x^{\omega\left(G_{\sigma_{1}, C}\right)-\left|\sigma_{1}\right|-k} y^{|C|+\omega\left(G_{\sigma_{1}, C}\right)-\left|\sigma_{1}\right|-k}
$$

for each $C \subseteq E\left(P_{k}\right)$ such that $\sigma_{1} \stackrel{C}{\longrightarrow} \sigma_{2}$, where $G_{\sigma_{1}, C}$ is defined in the proof of Lemma 3.1.

Here we are interested in the subsets $C \subseteq E\left(P_{k}\right)$ that contain the edge $e_{0}$ joining vertices $(1,1)$ and $(2,1)$. We define $U_{\sigma_{1} \sigma_{2}}$ to be the set given by

$$
U_{\sigma_{1} \sigma_{2}}=\left\{C \subseteq E\left(P_{k}\right) \mid e_{0} \in C, \sigma_{1} \stackrel{C}{\longrightarrow} \sigma_{2}\right\} .
$$

Now, the entry $\left(\sigma_{1}, \sigma_{2}\right)$ in $\Lambda_{k}^{\prime}$ is a non-zero polynomial if and only if $U_{\sigma_{1} \sigma_{2}}$ is non-empty. To prove that $D_{k}$ is a nonnegative matrix we need the following technical lemma.

Lemma 6.6. Let $k \geq 2$ and $\sigma_{1}, \sigma_{2} \in \mathcal{N C}_{k}$. If $U_{\sigma_{1}, \sigma_{2}}$ is non-empty, the value of the entry $\left(\sigma_{1}, \sigma_{2}\right)$ in the matrix $D_{k}$ is positive.

Proof. As mentioned, the entry $\left(\Lambda_{k}^{\prime}\right)_{\sigma_{1}, \sigma_{2}}$ is the sum of the terms

$$
x^{\omega\left(G_{\sigma_{1}, C}\right)-\left|\sigma_{1}\right|-k} y^{|C|+\omega\left(G_{\sigma_{1}, C}\right)-\left|\sigma_{1}\right|-k}
$$

over all $C$ in $U_{\sigma_{1}, \sigma_{2}}$. Every term, when evaluated at $x=1, y=0$ is either 1 or 0 . It is enough to prove that there exists $C$ in $U_{\sigma_{1}, \sigma_{2}}$ such that the corresponding term is 1 . For that, it suffices to prove that there exists $C$ in $U_{\sigma_{1}, \sigma_{2}}$ such that

$$
|C|+\omega\left(G_{\sigma_{1}, C}\right)-\left|\sigma_{1}\right|-k=0 .
$$


Suppose that there is no edge of $C$ belonging to a cycle of $G_{\sigma_{1}, C}$. Thus, the removal of any edge in $C$ increases the number of connected components by exactly 1 . By this argument it follows that $\omega\left(G_{\sigma_{1}, C} \backslash C\right)=\omega\left(G_{\sigma_{1}, C}\right)+|C|$. But by definition, $G_{\sigma_{1}, C} \backslash C$ has $\left|\sigma_{1}\right|+k$ connected components. Thus, if $C$ has no edge belonging to a cycle of $G_{\sigma_{1}, C}, C$ satisfies (6.6).

We now show that we can find such a $C$ in $U_{\sigma_{1}, \sigma_{2}}$. By hypothesis, $U_{\sigma_{1}, \sigma_{2}}$ is non-empty, so there exists $C_{0} \in U_{\sigma_{1}, \sigma_{2}}$. If $C_{0}$ has no edge belonging to a cycle of $G_{\sigma_{1}, C_{0}}$, we have the result. Suppose that $C_{0}$ has the edge $f_{0}$ in a cycle of $G_{\sigma_{1}, C_{0}}$. Note that such an edge can be taken to be different from $e_{0}$. Now consider $C_{1}=C_{0} \backslash f_{0}$. It is clear that the deletion of $f_{0}$ does not change the components of $G_{\sigma_{1}, C_{0}}$, so $C_{1} \in U_{\sigma_{1}, \sigma_{2}}$. We can now repeat the argument with $C_{1}$ instead of $C_{0}$. As this process is finite, we end up with a set $C$ with no edges in a cycle of $G_{\sigma_{1}, C}$ and the proof is complete.

Lemma 6.7. Let $k \geq 2$. The real matrix $D_{k}$ is nonnegative. Furthermore, the main diagonal entries of $D_{k}$ are positive.

Proof. The first statement follows from Lemma 6.6.

To see that the main diagonal entries of $D_{k}$ are positive, we just have to check, by Lemma 6.6, that $U_{\sigma, \sigma}$ is non-empty. But this is clear as the set $B_{I}$, given by

$$
B_{I}=\{(1, j) \sim(2, j) \mid 1 \leq j \leq k\}
$$

is always in $U_{\sigma, \sigma}$, for any $\sigma \in \mathcal{N C}_{k}$.

The same result is true if instead of the matrix $D_{k}$ we use $D_{k}^{\prime}$ but the proof involves so much notation that we decided to omit it. Instead we analyse the matrix $D_{k}^{\prime}$ for the particular values of $2 \leq k \leq 8$ that we need for one of the main results of this paper.

Proposition 6.8. Let $2 \leq k \leq 8$. If $\sigma, \gamma \in \mathcal{N C}_{k}$, then $\left(D_{k}^{\prime}\right)_{\sigma \gamma}$ is positive if and only if $\left(D_{k}\right)_{\sigma \gamma}$ is positive, and $\left(D_{k}^{\prime}\right)_{\sigma \gamma}$ is zero if and only if $\left(D_{k}\right)_{\sigma \gamma}$ is zero.

Proof. This was done by computing the matrices $D_{k}^{\prime}$ and $D_{k}$ for $2 \leq k \leq 8$ and comparing them entry by entry.

To get the full strength of the Perron-Frobenius Theorem we require the matrices $D_{k}$ and $D_{k}^{\prime}$ to be primitive matrices.

Definition 6.9. An $n \times n$ nonnegative real matrix $A$ is said to be primitive if it is irreducible and has only one eigenvalue of maximum modulus.

In view of Theorem 6.5 we need the following

Lemma 6.10. The digraph $\Gamma\left(D_{k}\right)$ is strongly connected, for $k \geq 2$.

Proof. We just give an sketch of the proof. To prove that $\Gamma\left(D_{k}\right)$ is strongly connected, we have to give for every pair $(\sigma, \gamma)$ of states a sequence $\sigma=\sigma_{0}, \ldots, \sigma_{p}=\gamma$, such that $U_{\sigma_{i}, \sigma_{i+1}}$ is non-empty for $0 \leq i \leq p-1$. 
Observe that we always have $U_{\sigma, \overline{1}} \neq \emptyset$, where $\overline{1}$ is the standard form in $\mathcal{S}_{k}$ with all the entries equal to 1 . Thus, it is enough to prove that for every $\gamma$ in $\mathcal{S}_{k}$, there is a sequence $\overline{1}=\sigma_{0}, \ldots, \sigma_{p}=\gamma$, such that $U_{\sigma_{i}, \sigma_{i+1}}$ is non-empty for $0 \leq i \leq p-1$. But this is clearly always possible for $p \leq k / 2$, and more than proving it, we have provided the reader with an example in Figure 4. The general construction can be easily deduced from this.

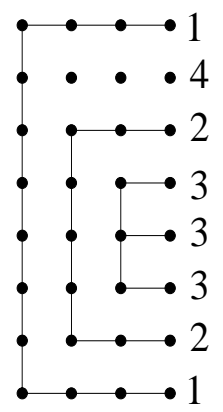

Figure 4: An example of the required construction in Lemma 6.10 with $k=8$ and $\gamma=(1,2,3,3,3,2,4,1)$.

Theorem 6.11. For $k \geq 2, D_{k}$ is a nonnegative primitive matrix. Also, for $2 \leq k \leq 8$, $D_{k}^{\prime}$ is a nonnegative primitive matrix.

Proof. From the previous lemma and Theorem 6.5, we know that $D_{k}$ is irreducible. Also Lemma 6.7 says that $D_{k}$ is nonnegative and that its main diagonal entries are positive. Thus, it follows from Lemma 8.5.5 and Theorem 8.5.2 in [6], that $D_{k}$ is primitive.

Let $2 \leq k \leq 8$. By Proposition 6.8 and the definition of $\Gamma\left(D_{k}^{\prime}\right)$, we get $\Gamma\left(D_{k}^{\prime}\right)=\Gamma\left(D_{k}\right)$. Thus $\Gamma\left(D_{k}^{\prime}\right)$ is strongly connected and again we obtain that $D_{k}^{\prime}$ is a nonnegative irreducible matrix with its main diagonal entries positive. Thus $D_{k}^{\prime}$ is primitive.

Theorem 6.12. Let $k \geq 2$, then

$$
\lim _{n \rightarrow \infty}\left(f\left(\mathcal{F}_{n}^{k-1}\right)\right)^{1 / n}=\rho\left(D_{k}\right) .
$$

Proof. We know by previous discussion that $f\left(\mathcal{F}_{m+1}^{k-1}\right)=a_{k}^{t} D_{k}^{m} \overrightarrow{1}$. Now, we apply the Perron-Frobenius Theorem, using the version in [6, Theorem 8.5.1], to obtain the following result.

$$
\begin{aligned}
\lim _{n \rightarrow \infty} \frac{f\left(\mathcal{F}_{n+1}^{k-1}\right)}{\rho\left(D_{k}\right)^{n}} & =\lim _{n \rightarrow \infty} \frac{a_{k}^{t} D_{k}^{n} \overrightarrow{1}}{\rho\left(D_{k}\right)^{n}} \\
& =a_{k}^{t}\left(\lim _{n \rightarrow \infty} \frac{D_{k}^{n}}{\rho\left(D_{k}\right)^{n}}\right) \overrightarrow{1} \\
& =a_{k}^{t} L \overrightarrow{1} .
\end{aligned}
$$

Where $L=z y^{t}, D_{k} z=\rho\left(D_{k}\right) z, D_{k}^{t} y=\rho\left(D_{k}\right) y, z>0, y>0$ and $z^{t} y=1$. 
Notice that $a_{k}>0$ and $L>0$ thus the real number $\theta=a_{k} L \overrightarrow{1}$ is strictly positive and we obtain

$$
\lim _{n \rightarrow \infty} \frac{f\left(\mathcal{F}_{n+1}^{k-1}\right)}{\rho\left(D_{k}\right)^{n}}=\theta>0 .
$$

Then

$$
\lim _{n \rightarrow \infty} f\left(\mathcal{F}_{n+1}^{k-1}\right)^{1 /(n+1)}=\lim _{n \rightarrow \infty} \theta^{1 /(n+1)} \rho\left(D_{k}\right)^{n /(n+1)}=\rho\left(D_{k}\right)
$$

A similar proof gives the following

Theorem 6.13. Let $2 \leq k \leq 8$, then

$$
\lim _{n \rightarrow \infty}\left(\alpha\left(\mathcal{F}_{n}^{k-1}\right)\right)^{1 / n}=\rho\left(D_{k}^{\prime}\right)
$$

Finally, using Theorem 6.1 and Theorem 6.2 together with the last two theorems we obtain the following

Corollary 6.14. For any fixed $k \geq 2$, we have that

$$
\rho\left(D_{k}\right)^{1 /(k-1)} \leq \lim _{n \rightarrow \infty} f(n)^{1 / n^{2}}
$$

and for $2 \leq k \leq 8$

$$
\rho\left(D_{k}^{\prime}\right)^{1 /(k-1)} \leq \lim _{n \rightarrow \infty} \alpha(n)^{1 / n^{2}}
$$

The programs indices.c and matrix2.c can generate the matrices $D_{k}$ and $D_{k}^{\prime}$ for small values of $k$. We use matlab to obtain the corresponding eigenvalues. Here the note of the last section applies and we consider these eigenvalues accurate up to the last decimal. For the following theorems we use the best values that we can compute, $\rho\left(D_{8}\right)$ and $\rho\left(D_{8}^{\prime}\right)$. As a note, the eigenvalue for $D_{3}^{\prime}$ is 10.405124837953 and then the lower bound using this value is $3.2256975738518 \approx \sqrt{(13+\sqrt{61}) / 2}$, which corresponds to a previous observation. We conclude this section with the following strengthening of the lower bounds given in [7].

\section{Theorem 6.15.}

$$
\begin{aligned}
& 3.64497565338648 \ldots \leq \lim _{n \rightarrow \infty} f(n)^{1 / n^{2}} \\
& 3.41358097503492 \ldots \leq \lim _{n \rightarrow \infty} \alpha(n)^{1 / n^{2}} .
\end{aligned}
$$

Just recently, S. C. Chang and R. Shrock found that $3.49 \leq \lim _{n \rightarrow \infty} \alpha(n)^{1 / n^{2}}[4]$. 


\section{Concluding remarks}

We have produced one additional result which we will just mention briefly.

Let $\beta(n)$ and $g(n)$ be, respectively, the number of totally cyclic orientations and the number of spanning connected subgraphs of $L_{n}$. We now that the limits

$$
\lim _{n \rightarrow \infty} \beta(n)^{1 / n^{2}}, \quad \lim _{n \rightarrow \infty} g(n)^{1 / n^{2}}
$$

exist. Let us show that,

$$
\lim _{n \rightarrow \infty} \beta(n)^{1 / n^{2}}=\lim _{n \rightarrow \infty} \alpha(n)^{1 / n^{2}}
$$

and that

$$
\lim _{n \rightarrow \infty} g(n)^{1 / n^{2}}=\lim _{n \rightarrow \infty} f(n)^{1 / n^{2}} .
$$

Recall from Section 2 that $\beta(n)=T\left(L_{n} ; 0,2\right)$ and that $g(n)=T\left(L_{n} ; 1,2\right)$. We also need the following facts. If $G$ is a plane graph and $G^{*}$ its dual graph, then $T\left(G^{*} ; x, y\right)=$ $T(G ; y, x)$ (see [3, Proposition 6.2.4]. Also, if $H$ is a subgraph of $G$ and $G$ has no loops, then the number of acyclic orientations of $G$ is at least that of $H$; this is because every acyclic orientation of $H$ can be extended to one of $G$. The same remark applies to the number of totally cyclic orientations if $G$ has no bridges.

Observe now that $L_{n}^{*}$ contains $L_{n-1}$ as a subgraph; in fact, $L_{n-1}$ is obtained from $L_{n}^{*}$ by deleting the vertex corresponding to the external face. Because of the previous observations we then have

$$
\beta(n)=T\left(L_{n}^{*} ; 2,0\right) \geq T\left(L_{n-1} ; 2,0\right)=\alpha(n-1) .
$$

And dually

$$
\alpha(n)=T\left(L_{n}^{*} ; 0,2\right) \geq T\left(L_{n-1} ; 0,2\right)=\beta(n-1) .
$$

This implies that

$$
\lim _{n \rightarrow \infty} \beta(n)^{1 / n^{2}}=\lim _{n \rightarrow \infty} \alpha(n)^{1 / n^{2}} .
$$

The proof of the second equality is very similar; it relies again on the formula $T\left(G^{*} ; x, y\right)=$ $T(G ; y, x)$, and the fact that the number of forests and the number of connected subgraphs are both increasing functions on subgraphs. Using a similar argument as before we get

$$
\begin{aligned}
& g(n)=T\left(L_{n}^{*} ; 2,1\right) \geq T\left(L_{n-1} ; 2,1\right)=f(n-1) \\
& f(n)=T\left(L_{n}^{*} ; 1,2\right) \geq T\left(L_{n-1} ; 1,2\right)=g(n-1) .
\end{aligned}
$$

The equality $\lim _{n \rightarrow \infty} g(n)^{1 / n^{2}}=\lim _{n \rightarrow \infty} f(n)^{1 / n^{2}}$ then follows.

We want to thank D. J. A. Welsh for the discussion which led to this paper. Also, we want to thank an anonymous referee for some very helpful comments. 


\section{References}

[1] N.L. Biggs, Colouring square lattice graphs, Null. London Math. Soc. 9 (1977), 54-56.

[2] A.V. Bakaev and V.I. Kabanovich, Series expansions for the $q$-colour problem on the square and cubic lattices, J. Phys. A:Math Gen 27 (1994) 6731-6739.

[3] T. Brylawski, J. Oxley, The Tutte Polynomial and its Applications, in Matroid Applications (ed. N. White), Cambridge Univ. Press (1992).

[4] S.C. Chang and R. Shrock, Acyclic orientation numbers for families of graphs, State Univ. of New York at Stony Brook preprint.

[5] N.J. Calkin and H.S. Wilf, The number of independent sets in a grid graph, SIAM J. Discrete Math 11 (1998), 54-60.

[6] R.A. Horn and C.R. Johnson, Matrix Analysis, Cambridge Univ. Press (1990).

[7] C. Merino and D.J.A. Welsh, Forests, colourings and acyclic orientations of the square lattice, Ann. of Combinatorics 3 (1999), 417-429.

[8] M. Noy and A. Ribó, Constructing recursive families of graphs. To appear.

[9] J.G. Oxley, Matroid Theory, Oxford Univ. Press (1992).

[10] R.P. Stanley, Enumerative combinatorics, Vol. 1 (corrected reprint of the 1986 original), Cambridge Univ. Press (1997).

[11] D.L. Vertigan and D.J.A. Welsh, The computational complexity of the Tutte plane: the bipartite case, Combin. Probab. Comput. 1 (1992), 181-187.

[12] D.L. Vertigan, D.J.A. Welsh and G.P. Whittle, Computing the Tutte polynomial of grid graphs is \#P-hard. To appear. 